Original Article

\title{
The Effect of Ground Tilt on the Lower Extremity Muscle Activity of Stroke Patients Performing Squat Exercises
}

\author{
Kyog-Il Ki, PT, MS ${ }^{1)}$, Jong-Duk Choi, PT, PhD²), Hyuk-Shin Cho, PT, MS ${ }^{3)}$ \\ 1) Department of Physical Therapy, Graduate School, Daejeon University, Republic of Korea \\ 2) Department of Physical Therapy, College of Natural Science, Daejeon University: 62 Daehak-ro, \\ Dong-gu, Daejeon 300-716, Republic of Korea \\ 3) Department of Physical Therapy, Wonkwang Health Science University, Republic of Korea
}

\begin{abstract}
Purpose] The purpose of this study was to determine the effect of ground tilt on the lower extremity muscle activity of stroke patients performing squat exercises. [Subjects] Fifteen hemiparetic patients volunteered to participate in this study. [Methods] The subjects performed squat exercises at three different ground tilt angles: $15^{\circ}$ plantar flexion, a neutral position, and $15^{\circ}$ dorsiflexion. A surface electromyogram (sEMG) was used to record the electromyographic activities of the leg extensor muscle in the vastus lateralis (VL), vastus medialis (VM), gastrocnemius lateralis (GL), and gastrocnemius medialis (GM). The sEMG activity was analyzed using a one-way repeated measures ANOVA and a post hoc Bonferroni correction. [Results] The results of this study are summarized as follows. Significant differences were noted for the VL and the GL when the angle of the ankle joint was between the $15^{\circ}$ plantar flexion and neutral positions during squat exercises involving the VL and when the angle of the ankle joint was between the neutral position and $15^{\circ}$ dorsiflexion during squat exercises involving the VM. [Conclusion] In this study, sEMG showed that the VL and GL changed significantly during squat exercises according to the ground tilt angle of hemiparetic patients. Therefore, squat exercises with different ground tilt angles can be used to improve VL and GL strength.

Key words: Electromyogram, Hemiplegic patient, Squat exercises
\end{abstract}

(This article was submitted Nov. 20, 2013, and was accepted Jan. 8, 2014)

\section{INTRODUCTION}

Patients with hemiparesis due to stroke tend to use the non-affected side of the body rather than the affected side ${ }^{1)}$. Therefore, muscle weakness in the upper and lower extremities of the affected side increases, and asymmetric body alignment and weight shift can occur ${ }^{2)}$. This leads to difficulty in activities of daily living ${ }^{3)}$. For hemiparetic patients, improvement of independent walking ability is an important criterion for functional recovery, as well as one of the aims of treatment ${ }^{4,5}$.

The affected side lower extremity muscle weakness in hemiparetic patients plays an important role in the restriction of walking ability ${ }^{6,7)}$. Muscle strengthening exercises for the lower extremities, especially strengthening the extensor muscle in the knee joint, is very effective in the improvement of balance during walking, walking speed, and walking distance ${ }^{8)}$.

Among the lower extremity muscles, the quadriceps

*Corresponding author. Jong-Duk Choi (E-mail: choidew@ dju.kr)

C2014 The Society of Physical Therapy Science. Published by IPEC Inc. This is an open-access article distributed under the terms of the Creative Commons Attribution Non-Commercial No Derivatives (by-ncnd) License $<$ http://creativecommons.org/licenses/by-nc-nd/3.0/>. femoris muscle is important for dynamic stability in a standing position, while the gastrocnemius muscle is very important in the generation of vertical ground reaction force for push-off during walking ${ }^{9,10)}$. Therefore, musclestrengthening exercises using body weight have typically been used to improve muscle strength in the lower extremities in clinical practice. Among them, squatting is the most appropriate exercise method to strengthen the quadriceps femoris, making it ideal to strengthen lower extremity muscles for patients with functional problems due to damage to the neuromusculoskeletal system ${ }^{5}$.

Tang et al. reported that the vastus medialis muscle improved after using closed kinetic chain (CKC) exercises, such as squatting ${ }^{11)}$. In particular, squatting performed at a knee joint angle between $45^{\circ}$ and $60^{\circ}$ showed the most effective result. However, most studies have been done with healthy people or athletes. Few case studies have been done with hemiparetic patients. Furthermore, very few studies have been done on changes in muscle activity of the lower extremities in the squatting position in conjunction with changes in ankle joint angle in a variety of ground environments.

The purpose of this study was to examine the effect of changes in ground tilt angle during squatting on the affected lower extremity muscle activity of hemiparetic patients, and the results of this study would be used as foundational 
data for rehabilitation programs for lower extremity muscle strengthening in hemiparetic patients.

\section{SUBJECTS AND METHODS}

Chronic hemiparetic patients $(n=15)$ with at least 6 months elapsed after being diagnosed with stroke participated in this study after giving informed consent. For the criteria to select the subjects, three main points were considered. The selected subjects had to be able to walk independently with assistive devices and had to have a score of more than 5 on the walk item of the Functional Independence Measure (FIM), they had to be able to understand and perform the research instructions with a score of more than 25 on the Mini-Mental State Examination (MMSE), and they had to have no orthopedic disease in both lower extremities (Table 1). All of the protocols used in this study were approved by the University of Daejeon. Before participation, the procedures, risk, and benefits were explained to all the participants, who gave their informed consent. The participants' rights were protected according to the guidelines of the University of Daejeon.

To measure extensor muscle activity in the affected lower extremity, a QEMG-4 (LXM3204, Laxtha, Daejeon, South Korea), a 4-channel surface electromyogram (sEMG) system, was used. The collected data were then analyzed with the Telescan 2.89 electromyogram software (Laxtha, Daejeon, South Korea). For the surface electrode, an Ag/ $\mathrm{AgCl}$ electrode (3M, St. Paul, MN, USA) with a sampling rate of $1024 \mathrm{~Hz}$ was used. Signals were processed through a band-pass filter of $20-450 \mathrm{~Hz}$ and a notch filter of $60 \mathrm{~Hz}$. For the sEMG electrode position, electrodes were attached to the vastus medialis, vastus lateralis, gastrocnemius medialis, and gastrocnemius lateralis with the proposed SENIAM project method (Surface Electromyography for the Non-Invasive Assessment of Muscles, 2005). During squatting, the sEMG signal was collected in five-second segments, and the measurement was done three times for each position. The sEMG data obtained in the first and last one second were removed, and only the data for the three seconds between the first and last seconds were transformed into root mean square (RMS) values and recorded.

The experiment was done at three positions $\left(15^{\circ}\right.$ ankle joint plantar flexion, neutral, and $15^{\circ}$ dorsiflexion) and using our own manufactured quadriceps board. For squatting, the knee joint was positioned at $45^{\circ}$ of flexion, using a goniometer while the legs were placed shoulder-width distance from each other and the hands were placed freely. To shift weight to the affected side during squatting, a posture mirror, on which a line was drawn, was used. A Sufficient amount of practice was performed to ensure that the same experimental posture would be used during measurement. Measurement in each experiment was done three times, and the order of the experiment was assigned randomly. To reduce muscle fatigue, a one-minute rest break was given between the experiments.

SPSS version 18.0 for Windows was used for statistical analysis. For general characteristics of the subject, descriptive statistics was used. One-way repeated measures ANOVA was used to compare the lower extremity muscle activity according to the ground tilt angle, and the post hoc Bonferroni correction was applied. The level of significance was set as $\alpha=0.05$.

\section{RESULTS}

The muscle activity in the affected lower extremity during squatting according to the three positions $\left(15^{\circ}\right.$ ankle joint plantar flexion, neutral, and $15^{\circ}$ dorsiflexion) is shown

Table 1. General characteristics of the subjects

\begin{tabular}{lc}
\hline Characteristics & $(\mathrm{n}=15)$ \\
\hline Gender & \\
Male & $8(53)^{\mathrm{a}}$ \\
Female & $7(47)$ \\
Age $(\mathrm{yr})$ & $67.13 \pm 13.30^{\mathrm{b}}$ \\
Height $(\mathrm{cm})$ & $161.47 \pm 5.32$ \\
Weight $(\mathrm{kg})$ & $59.67 \pm 9.06$ \\
BMI $\left(\mathrm{kg} / \mathrm{m}^{2}\right)^{\mathrm{c}}$ & $22.80 \pm 2.64$ \\
Stroke type & \\
Infarction & $8(53)$ \\
Hemorrhage & $7(47)$ \\
Paretic side & \\
Left & $9(60)$ \\
Right & $6(40)$ \\
MAS ${ }^{\mathrm{d}}$ & \\
Grade 0 & $0(0)$ \\
Grade 1 & $12(80)$ \\
Grade 1+ & $3(20)$ \\
Duration (mo.) & $17.73 \pm 12.83$ \\
MMSE-K & $27.12 \pm 2.81$ \\
\hline${ }^{\mathrm{a}}$ (\%), ${ }^{\mathrm{b}}$ Mean \pm SD, ${ }^{\mathrm{c}} \mathrm{BMI}:$ & body mass index, \\
$\mathrm{d}$ MAS: Modified Ashworth Scale, ${ }^{\mathrm{e}} \mathrm{MMSE}-\mathrm{K}:$ \\
Mini-Mental State Examination-Korea
\end{tabular}

Table 2. Comparison of electromyography activation according to ground tilt angle $(\mathrm{n}=15)$

\begin{tabular}{lccc}
\hline \multirow{2}{*}{ Muscle } & \multicolumn{3}{c}{ Ground tilt angle } \\
\cline { 2 - 4 } & $-15^{\circ}$ & $0^{\circ}$ & $15^{\circ}$ \\
\hline Vastus medialis & $51.80 \pm 31.06^{\mathrm{a}}$ & $48.25 \pm 28.79$ & $46.74 \pm 27.97$ \\
Vastus lateralis* & $62.67 \pm 34.45$ & $54.89 \pm 29.41$ & $54.74 \pm 30.83$ \\
Gastrocnemius medialis & $21.14 \pm 7.06$ & $18.65 \pm 4.14$ & $19.41 \pm 10.61$ \\
Gastrocnemius lateralis* & $29.09 \pm 9.47$ & $28.21 \pm 10.50$ & $22.81 \pm 8.39$ \\
\hline
\end{tabular}

${ }^{\mathrm{a}}$ Mean $(\% \mathrm{RMS}) \pm \mathrm{SD},{ }^{*} \mathrm{p}<0.05$ 
in Table 2. For the vastus medialis, the muscle activity during squatting was the highest at $51.80 \%$ for $15^{\circ}$ plantar flexion and the lowest at $46.74 \%$ for $15^{\circ}$ dorsiflexion. However, no statistical significance was shown $(p>0.05)$. For the vastus lateralis, the highest muscle activity was $52.67 \%$ at $15^{\circ}$ plantar flexion and the lowest was $54.74 \%$ at $15^{\circ}$ dorsiflexion. These results were statistically significant $(\mathrm{p}<0.05)$. For the gastrocnemius medialis, the highest muscle activity was $21.14 \%$ at $15^{\circ}$ plantar flexion, and the lowest was $18.65 \%$ at $0^{\circ}$ neutral position. However, no statistical significance was shown $(\mathrm{p}>0.05)$. For the gastrocnemius lateralis, the highest muscle activity was $29.09 \%$ at $15^{\circ}$ plantar flexion and the lowest was $22.81 \%$ at $15^{\circ}$ dorsiflexion. These results were statistically significant $(\mathrm{p}<0.05)$.

\section{DISCUSSION}

CKC exercises, like squatting, can cause co-contraction of the quadriceps femoris and hamstring and reduce anterior-posterior tibiofemoral translation. Since squatting facilitates selective contraction of the vastus medialis compared with open kinetic chain (OKC) exercise, it also has been used frequently for knee joint rehabilitation ${ }^{11)}$.

The alignment and movement of the patella is determined by the amount and direction of force applied to the soft tissues around the knee joint; in particular, the vastus medialis acts as a medial stabilizing muscle for the patel$\mathrm{la}^{12)}$. Contraction of the vastus medialis and rectus femoris can cause medial tilting and lateral rotation of the patella ${ }^{13)}$. The function of the patella is lateral tilt and rotation during knee extension from $0^{\circ}$ to $20^{\circ}$ in the $\mathrm{CKC}^{14)}$ and lateral tilt and rotation during squatting from $0^{\circ}$ to $90^{\circ 15)}$. Furthermore, the patella is moved medially during the initial period of squatting between $0^{\circ}$ and $20^{\circ}$ and then laterally above a knee flexion angle of about $45^{\circ 16}$ ).

To determine the patella movement and the Q-angle during knee joint movement, analysis was done using a threedimensional motion analysis system, and the results showed that the in addition to the increase in Q-angle, the patella was tilted and rotated laterally up to $45^{\circ}$ of knee flexion ${ }^{15}$. Therefore, it caused increasing activation of the vastus lateralis, thereby producing lateral tilt of the patella. Although measurement of the lateral tilt of the patella was not done in this study, a significant change in the vastus lateralis occurred in the above study.

Experiments with healthy subjects showed that when the ankle was moved, the lack of stability of the knee joint caused co-contraction of the vastus medialis and vastus lateralis ${ }^{11)}$. When a squat extension exercise was performed on flat ground, extension occurred through the contraction in the quadriceps femoris. However, when the ankle joint tilt was applied to the heel, the activation of both the quadriceps femoris and knee extension increased. Knee extension and ankle plantar flexion were used at the same time to lift the center of gravity, and squatting might be facilitated by reducing fatigue of the quadriceps femoris through activation of the gastrocnemius ${ }^{16)}$.

The results of the above study were similar to the measurement results of this study in that activation of the af- fected gastrocnemius showed the highest at $15^{\circ}$ plantar flexion during squatting. However, Cook et al. showed that the effect on the gastrocnemius was at the minimum during squatting at $25^{\circ}$ to $30^{\circ}$ on a descending ramp ${ }^{17)}$. However, the significant change in the affected gastrocnemius lateralis during squatting, performed when the ground tilt angle was $15^{\circ}$ plantar flexion, was thought to be affected by the increase in activation of the vastus lateralis and the lateral tilting of the patella up to $45^{\circ}$ of knee joint flexion in the Q-angle analysis and during patella movement, as reported by Wilson et $\mathrm{al}^{15}$.

Based on the previous studies showing that changes in ground tilt angle during squatting can influence stability and lower extremity muscle activity, this study also compared the sEMG activation of the quadriceps femoris and gastrocnemius in hemiparetic patients. More activation of the vastus lateralis and gastrocnemius lateralis was found in the squatting position when the ankle joint was in the plantar flexion condition, while inhibition was found in the squatting position when the ankle joint was in the dorsiflexion condition. Therefore, the results suggest that it is more effective for a stroke patient in the initial phase, as characterized by having the problem of spasticity of the ankle plantar flexor, to apply squatting on the ground in the dorsiflexion condition rather than on flat ground for the purpose of inhibition. On the other hand, it is more effective to apply squatting on the ground in the ankle joint plantar flexion condition rather than on flat ground to increase overall muscle strength of the lower extremities.

\section{REFERENCES}

1) Campbell FM, Ashburn AM, Pickering RM, et al.: Head and pelvic movements during a dynamic reaching task in sitting: implications for physical therapists. Arch Phys Med Rehabil, 2001, 82: 1655-1660. [Medline] [CrossRef]

2) Dickstein R, Nissan M, Pillar T, et al.: Foot-ground pressure pattern of standing hemiplegic patients. Major characteristics and patterns of improvement. Phys Ther, 1984, 64: 19-23. [Medline]

3) Werner C, Von Frankenberg S, Treig T, et al.: Treadmill training with partial body weight support and an electromechanical gait trainer for restoration of gait in subacute stroke patients: a randomized crossover study. Stroke, 2002, 33: 2895-2901. [Medline] [CrossRef]

4) Patterson SL, Forrester LW, Rodgers MM, et al: Determinants of walking function after stroke: differences by deficit severity. Arch Phys Med Rehabil, 2007, 88: 115-119. [Medline] [CrossRef]

5) Ng MF, Tong RK, Li LS: A pilot study of randomized clinical controlled trial of gait training in subacute stroke patients with partial body-weight support electromechanical gait trainer and functional electrical stimulation: six-month follow-up. Stroke, 2008, 39: 154-160. [Medline] [CrossRef]

6) Bohannon RW: Recovery and correlates of trunk muscle strength after stroke. Int J Rehabil Res, 1995, 18: 162-167. [Medline] [CrossRef]

7) Engardt $M$, Knutsson $E$, Jonsson $M$, et al.: Dynamic muscle strength training in stroke patients: effects on knee extension torque, electromyographic activity, and motor function. Arch Phys Med Rehabil, 1995, 76: 419-425. [Medline] [CrossRef]

8) Salbach NM, Mayo NE, Wood-Dauphinee S, et al.: A task-orientated intervention enhances walking distance and speed in the first year post stroke: a randomized controlled trial. Clin Rehabil, 2004, 18: 509-519. [Medline] [CrossRef]

9) Moxley Scarborough D, Krebs DE, Harris BA: Quadriceps muscle strength and dynamic stability in elderly persons. Gait Posture, 1999, 10: 10-20. [Medline] [CrossRef]

10) Carlsöö S, Dahlöf AG, Holm J: Kinetic analysis of the gait in patients with hemiparesis and in patients with intermittent claudication. Scand J Rehabil Med, 1974, 6: 166-179. [Medline] 
11) Tang SF, Chen CK, Hsu R, et al.: Vastus medialis obliquus and vastus lateralis activity in open and closed kinetic chain exercises in patients with patellofemoral pain syndrome: an electromyographic study. Arch Phys Med Rehabil, 2001, 82: 1441-1445. [Medline] [CrossRef]

12) Amis AA, Senavongse W, Bull AM: Patellofemoral kinematics during knee flexion-extension: an in vitro study. J Orthop Res, 2006, 24: 2201 2211. [Medline] [CrossRef]

13) Lin F, Wilson NA, Makhsous M, et al.: In vivo patellar tracking induced by individual quadriceps components in individuals with patellofemoral pain. J Biomech, 2010, 43: 235-241. [Medline] [CrossRef]

14) Lin F, Makhsous $\mathrm{M}$, Chang $\mathrm{AH}$, et al.: In vivo and noninvasive six degree of freedom patellar tracking during voluntary knee movement. Clin Bio- mech (Bristol, Avon), 2003, 18: 401-409. [Medline] [CrossRef]

15) Wilson NA, Press JM, Koh JL, et al.: In vivo noninvasive evaluation of abnormal patellar tracking during squatting in patients with patellofemoral pain. J Bone Joint Surg Am, 2009, 91: 558-566. [Medline] [CrossRef]

16) Coughlin KM, Incavo SJ, Churchill DL, et al.: Tibial axis and patellar position relative to the femoral epicondylar axis during squatting. J Arthroplasty, 2003, 18: 1048-1055. [Medline] [CrossRef]

17) Cook JL, Khan KM, Kiss ZS, et al.: Reproducibility and clinical utility of tendon palpation to detect patellar tendinopathy in young basketball players. Victorian Institute of Sport tendon study group. Br J Sports Med, 2001, 35: 65-69. [Medline] [CrossRef] 